\title{
Article \\ New Criteria for Oscillation of Half-Linear Differential Equations with $p$-Laplacian-like Operators
}

\author{
Omar Bazighifan ${ }^{1,2,+}$ (D) F. Ghanim ${ }^{3, *,+} \mathbb{D}$, Jan Awrejcewicz ${ }^{4, *}++^{(D)}$, Khalil S. Al-Ghafri ${ }^{5,+}$ (D) \\ and Maryam Al-Kandari ${ }^{6,+}$
}

1 Section of Mathematics, International Telematic University Uninettuno, Corso Vittorio Emanuele II, 39, 00186 Rome, Italy; o.bazighifan@gmail.com

2 Department of Mathematics, Faculty of Science, Hadhramout University, Hadhramout 50512, Yemen

3 Department of Mathematics, College of Sciences, University of Sharjah, Sharjah 27272, United Arab Emirates

4 Department of Automation, Biomechanics and Mechatronics, Lodz University of Technology, 1/15 Stefanowskiego St., 90-924 Lodz, Poland

5 University of Technology and Applied Sciences, P.O. Box 14, Ibri 516, Oman; khalil.ibr@cas.edu.om

6 Department of Mathematics, Kuwait University, P.O. Box 5969, Safat 13060, Khaldiyah City, Kuwait; maryam@sci.kuniv.edu.kw

* Correspondence: fgahmed@sharjah.ac.ae (F.G.); jan.awrejcewicz@p.lodz.pl (J.A.)

+ These authors contributed equally to this work.

check for

updates

Citation: Bazighifan, O.; Ghanim, F.; Awrejcewicz, J., Al-Ghafri, K.S.;

Al-Kandari, M. New Criteria for Oscillation of Half-Linear Differential Equations with $p$-Laplacian-like Operators. Mathematics 2021, 9, 2584. https://doi.org/10.3390/ math9202584

Academic Editor: Ioannis G. Stratis

Received: 16 September 2021

Accepted: 10 October 2021

Published: 14 October 2021

Publisher's Note: MDPI stays neutral with regard to jurisdictional claims in published maps and institutional affiliations.

Copyright: (C) 2021 by the authors. Licensee MDPI, Basel, Switzerland. This article is an open access article distributed under the terms and conditions of the Creative Commons Attribution (CC BY) license (https:/ / creativecommons.org/licenses/by/ $4.0 /)$.
Abstract: In this paper, new oscillatory properties for fourth-order delay differential equations with $p$-Laplacian-like operators are established, using the Riccati transformation and comparison method. Moreover, our results are an extension and complement to previous results in the literature. We provide some examples to examine the applicability of our results.

Keywords: fourth-order; delay differential equations; oscillation; $p$-Laplacian-like operator

MSC: 34C10; 34K11

\section{Introduction}

Delay differential equations arise in a variety of phenomena, including mixing liquids, economics problems, biology, medicine, physics, engineering and automatic control problems, as well as vibrational motion in flight and to explain human self-balancing; see [1,2].

The aim of this article is to study the oscillation conditions of differential equations with $p$-Laplacian-like operators:

$$
\left(\eta(\imath)\left|w^{\prime \prime \prime}(\imath)\right|^{p_{1}-2} w^{\prime \prime \prime}(\imath)\right)^{\prime}+\sum_{i=1}^{j} \vartheta_{i}(\imath)\left|w\left(\phi_{i}(\imath)\right)\right|^{p_{2}-2} w\left(\phi_{i}(\imath)\right)=0
$$

and

$$
\left(\eta(\imath)\left|w^{\prime \prime \prime}(\imath)\right|^{p_{1}-2} w^{\prime \prime \prime}(\imath)\right)^{\prime}+\beta(\imath)\left|w^{\prime \prime \prime}(\imath)\right|^{p_{1}-2} w^{\prime \prime \prime}(\imath)+\sum_{i=1}^{j} \vartheta_{i}(\imath)\left|w\left(\phi_{i}(\imath)\right)\right|^{p_{2}-2} w\left(\phi_{i}(\imath)\right)=0 .
$$

Throughout this work, we suppose the following hypotheses:

$\left(H_{1}\right) p_{i}>1, i=1,2$ are real numbers and $j \geq 1$,

$\left(H_{2}\right) \eta, \beta, \vartheta_{i} \in C\left(\left[\imath_{0}, \infty\right),[0, \infty)\right), \eta(\imath)>0, \vartheta_{i}(\imath)>0, \eta^{\prime}(\imath)+\beta(\imath) \geq 0, \phi_{i}(\imath) \in C\left(\left[\imath_{0}, \infty\right), \mathbb{R}\right), \phi_{i}(\imath) \leq \imath$, $\lim _{l \rightarrow \infty} \phi_{i}(l)=\infty, i=1,2, \ldots, j$.

Moreover, we study (1) under the condition

$$
\int_{l_{0}}^{\infty} \frac{1}{\eta^{1 / p_{1}-1}(s)} \mathrm{d} s=\infty
$$


and (2) under the condition

$$
\int_{l_{0}}^{\infty}\left[\frac{1}{\eta(s)} \exp \left(-\int_{l_{0}}^{s} \frac{\beta(x)}{\eta(x)} d x\right)\right]^{1 / p_{1}-1} d s=\infty .
$$

Definition 1. A solution $w$ of (1) and (2) is said to be non-oscillatory if it is ultimately positive or negative; otherwise, it is said to be oscillatory.

Definition 2. Equations (1) and (2) are called oscillatory if all of their solutions are oscillatory.

\section{Literature Review}

During recent decades, there is an ongoing interest in obtaining several sufficient conditions for the oscillatory behavior of the solutions of different kinds of differential equations, especially their oscillation and asymptote. Dzrina and Jadlovska [3], Bohner et al. [4] and Baculikova [5] developed approaches and techniques for studying oscillatory properties in order to improve the oscillation criteria of second-order differential equations with delay/middle terms. Baculikova et al. [6] and Grace et al. [7] also extended this evolution to delay differential equations. Therefore, there are many studies on the oscillation criteria of different orders of some differential equations with $p$-Laplacianlike operators; see [8,9]. With regard to their practical importance, the oscillation and asymptote of delay differential equations have been studied extensively in recent decades; see [10-28].

Li et al. [8] considered the oscillation for the delay equation

$$
\left(\eta(\imath)\left|z^{\prime \prime \prime}(\imath)\right|^{p-2} z^{\prime \prime \prime}(\imath)\right)^{\prime}+\sum_{i=1}^{j} \vartheta_{i}(\imath) w\left(\phi_{i}(\imath)\right)=0,
$$

where $\phi_{i}(\imath) \leq \imath$, and they used the Riccati technique to find oscillation conditions for this equation. Park et al. [26] studied the asymptotic properties of the solutions of the delay equation

$$
\left(\eta(\imath)\left|w^{(\kappa-1)}(\iota)\right|^{p-2} w^{(\kappa-1)}(\iota)\right)^{\prime}+\vartheta(\imath) g(w(\phi(\iota)))=0,
$$

where $\phi(\imath) \leq \imath, \kappa$ is even, and they used the integral average technique to obtain some oscillation results for this equation under the condition

$$
\int_{v_{0}}^{\infty} \frac{1}{\eta^{1 /(p-1)}(s)} \mathrm{d} s=\infty
$$

Zhang et al. [9] discussed the equation

$$
\left(\eta(\imath)\left(w^{(\kappa-1)}(\imath)\right)^{p-1}\right)^{\prime}+\beta(\imath)\left|w^{\prime \prime \prime}(\imath)\right|^{p_{1}-2} w^{\prime \prime \prime}(\imath)+\vartheta(\imath) f(w(\phi(\imath)))=0 .
$$

The purpose of this paper is to continue the authors' work $[13,14]$.

Many researchers have used the comparison method to find oscillation conditions for this equation.

The authors in $[8,9,26]$ used the integral average and comparison techniques that differ from the approach used in this article. Their approach is based on using the comparison technique to reduce Equations (1) and (2) into a first-order equation, while our article is based on using the Riccati technique to reduce Equations (1) and (2) into a first-order inequality to find more effective oscillation conditions for Equations (1) and (2).

Motivated by the reasons mentioned above, in this paper, we extend the results using Riccati and comparison techniques under (3) and (4). These results contribute to adding some important conditions that were previously studied in the subject of oscillation of differential equations with neutral terms. To prove our main results, we give some examples.

We shall establish asymptotic properties for (2) by converting into the form (1). It is not difficult 
to see that

$$
\begin{aligned}
\frac{1}{\zeta_{l_{0}}(\imath)} \frac{\mathrm{d}}{\mathrm{d} \imath}\left(\mu(\imath) \eta(\imath)\left(w^{\prime \prime \prime}(\imath)\right)^{p_{1}-1}\right) & =\frac{1}{\zeta_{l_{0}}(\imath)}\left(\zeta_{l_{0}}(\imath)\left(\eta(\imath)\left(w^{\prime \prime \prime}(\imath)\right)^{p_{1}-1}\right)^{\prime}\right) \\
& +\frac{1}{\zeta_{l_{0}}(\imath)}\left(\zeta_{\iota_{0}}^{\prime}(\imath) \eta(\imath)\left(w^{\prime \prime \prime}(\imath)\right)^{p_{1}-1}\right) \\
& =\left(\eta(\imath)\left(w^{\prime \prime \prime}(\imath)\right)^{p_{1}-1}\right)^{\prime}+\frac{\zeta_{l_{0}}^{\prime}(\imath)}{\zeta_{l_{0}}(\imath)} \eta(\imath)\left(w^{\prime \prime \prime}(\imath)\right)^{p_{1}-1}, \\
& =\left(\eta(\imath)\left(w^{\prime \prime \prime}(\imath)\right)^{p_{1}-1}\right)^{\prime}+\beta(\imath)\left(w^{\prime \prime \prime}(\imath)\right)^{p_{1}-1},
\end{aligned}
$$

which with (2) gives

$$
\left(\zeta_{\iota_{0}}(\imath) \eta(\imath)\left(w^{\prime \prime \prime}(\imath)\right)^{p_{1}-1}\right)^{\prime}+\zeta_{l_{0}}(\imath) \sum_{i=1}^{j} \vartheta_{i}(\imath) w^{p_{2}-1}\left(\phi_{i}(\imath)\right)=0 .
$$

To prove the main results, we present some lemmas:

Lemma 1. In [15], if the function $w$ satisfies $w^{(i)}(\imath)>0, i=0,1, \ldots, n$, and $w^{(n+1)}(\imath)<0$, then

$$
\frac{w(\imath)}{\imath^{n} / n !} \geq \frac{w^{\prime}(\imath)}{\imath^{n-1} /(n-1) !}
$$

Lemma 2. In [16], let $h \in C^{n}\left(\left[\imath_{0}, \infty\right),(0, \infty)\right)$. Suppose that $h^{(n)}(\imath)$ is of a fixed sign, on $\left[\imath_{0}, \infty\right), h^{(n)}(l)$, not identically zero and that there exists an $\imath_{1} \geq \imath_{0}$ such that, for all $\imath \geq \imath_{1}$,

$$
h^{(n-1)}(\imath) h^{(n)}(\imath) \leq 0 .
$$

If we have $\lim _{\imath \rightarrow \infty} h(\imath) \neq 0$, then there exists an $\imath_{\lambda} \geq \imath_{0}$ such that

$$
h(\imath) \geq \frac{\lambda}{(n-1) !} \imath^{n-1}\left|h^{(n-1)}(\imath)\right|
$$

for all $\lambda \in(0,1)$ and $\iota \geq \imath_{\lambda}$.

Lemma 3. In [17], let $\kappa$ be a ratio of two odd numbers, $V>0$ and $U$, that are constants. Then,

$$
U u-V u^{(\kappa+1) / \kappa} \leq \frac{\kappa^{\kappa}}{(\kappa+1)^{\kappa+1}} U^{\kappa+1} V^{-\kappa} .
$$

\section{Oscillation Criteria}

For convenience, we denote

$$
\begin{gathered}
R(\imath):=\int_{\imath}^{\infty}\left(\frac{1}{\eta(x)} \int_{x}^{\infty} \sum_{i=1}^{j} \vartheta_{i}(s) \mathrm{d} s\right)^{1 /\left(p_{1}-1\right)} \mathrm{d} x, \\
\widetilde{R}(\imath):=\mu_{2}^{\left(p_{2}-1\right) /\left(p_{1}-1\right)} \int_{\imath}^{\infty}\left(\frac{1}{\eta(x)} \int_{x}^{\infty} \sum_{i=1}^{j} \vartheta_{i}(s)\left(\frac{\phi_{i}(s)}{s}\right)^{p_{2}-1} \mathrm{~d} s\right)^{1 /\left(p_{1}-1\right)} \mathrm{d} x, \\
\zeta_{\iota_{0}}(\imath):=\exp \left(\int_{\imath_{0}}^{\imath} \frac{\beta(x)}{\eta(x)} \mathrm{d} x\right)
\end{gathered}
$$

and

$$
\widehat{R}(\imath):=\mu_{2}^{\left(p_{2}-1\right) /\left(p_{1}-1\right)} \int_{\imath}^{\infty}\left(\frac{1}{\eta(x) \zeta_{\iota_{0}}(l)} \int_{x}^{\infty} \zeta_{\imath_{0}}(\imath) \sum_{i=1}^{j} \vartheta_{i}(s)\left(\frac{\phi_{i}(s)}{s}\right)^{p_{2}-1} \mathrm{~d} s\right)^{1 /\left(p_{1}-1\right)} \mathrm{d} x,
$$

where $\mu_{2} \in(0,1)$. 
Lemma 4. Let (3) hold. If $w$ is an eventually positive solution of (1), then $w^{\prime}>0$ and $w^{\prime \prime \prime}>0$.

Proof. The proof is obvious and therefore is omitted.

Theorem 1. If the equation

$$
x^{\prime}(\imath)+\frac{\lambda^{p_{2}-1}}{6^{p_{2}-1}} \frac{\sum_{i=1}^{j} \vartheta_{i}(\imath) \phi_{i}^{3\left(p_{2}-1\right)}(\imath)}{\eta^{\left(p_{2}-1\right) /\left(p_{1}-1\right)}\left(\phi_{i}(\imath)\right)} x^{\left(p_{2}-1\right) /\left(p_{1}-1\right)}\left(\phi_{i}(\imath)\right)=0
$$

is oscillatory, then (1) is oscillatory.

Proof. Assume that (1) has a nonoscillatory solution in $\left[\imath_{0}, \infty\right)$. Then, there exists a $\imath_{1} \geq \imath_{0}$ such that $w(\imath)>0$ and $w\left(\phi_{i}(\imath)\right)>0$ for $\imath \geq \imath_{1}$. Let

$$
x(\imath):=\eta(\imath)\left(w^{\prime \prime \prime}(\imath)\right)^{p_{1}-1}>0[\text { from Lemma } 4] .
$$

It is known that

$$
\left|w^{\prime \prime \prime}(\imath)\right|^{p_{1}-2} w^{\prime \prime \prime}(\imath)=\left(w^{\prime \prime \prime}(\imath)\right)^{p_{1}-1} \text { and }\left|w\left(\phi_{i}(\imath)\right)\right|^{p_{2}-2} w\left(\phi_{i}(\imath)\right)=w^{p_{2}-1}\left(\phi_{i}(\imath)\right) .
$$

From (1) and (7), we obtain

$$
x^{\prime}(\imath)+\sum_{i=1}^{j} \vartheta_{i}(\imath) w^{p_{2}-1}\left(\phi_{i}(\imath)\right)=0 .
$$

Since $w$ is positive and increasing, we see $\lim _{l \rightarrow \infty} w(\imath) \neq 0$. So, using Lemma 2 , we find

$$
w^{p_{2}-1}\left(\phi_{i}(\imath)\right) \geq \frac{\lambda^{p_{2}-1}}{6^{p_{2}-1}} \phi_{i}^{3\left(p_{2}-1\right)}(\imath)\left(w^{\prime \prime \prime}\left(\phi_{i}(\imath)\right)\right)^{p_{2}-1},
$$

for all $\lambda \in(0,1)$. By (8) and (9), we see that

$$
x^{\prime}(\imath)+\frac{\lambda^{p_{2}-1}}{6^{p_{2}-1}} \sum_{i=1}^{j} \vartheta_{i}(\imath) \phi_{i}^{3\left(p_{2}-1\right)}(\imath)\left(w^{\prime \prime \prime}\left(\phi_{i}(\imath)\right)\right)^{p_{2}-1} \leq 0 .
$$

Thus, $x$ is a positive solution of the inequality

$$
x^{\prime}(\imath)+\frac{\lambda^{p_{2}-1}}{6^{p_{2}-1}} \frac{\sum_{i=1}^{j} \vartheta_{i}(\imath) \phi_{i}^{3\left(p_{2}-1\right)}(\imath)}{\eta^{\left(p_{2}-1\right) /\left(p_{1}-1\right)}\left(\phi_{i}(\imath)\right)} x^{\left(p_{2}-1\right) /\left(p_{1}-1\right)}\left(\phi_{i}(\imath)\right) \leq 0 .
$$

By using Theorem 1 [22], we find that (6) also has a positive solution, which is a contradiction. The proof is complete.

Corollary 1. Assume that $p_{2}=p_{1}$ and (3) holds. If

$$
\liminf _{\imath \rightarrow \infty} \int_{\phi(\imath)}^{\imath} \frac{\lambda^{p_{2}-1}}{6^{p_{2}-1}} \frac{\sum_{i=1}^{j} \vartheta_{i}(s) \phi_{i}^{3\left(p_{2}-1\right)}(s)}{\eta^{\left(p_{2}-1\right) /\left(p_{1}-1\right)}\left(\phi_{i}(s)\right)} \mathrm{d} s>\frac{1}{\mathrm{e}^{\prime}}
$$

then (1) is oscillatory.

Lemma 5. If

$$
\int_{\imath_{0}}^{\infty}\left(M^{p_{2}-p_{1}} \varsigma(s) \sum_{i=1}^{j} \vartheta_{i}(s) \frac{\phi_{i}^{3\left(p_{2}-1\right)}(s)}{s^{3 \kappa}}-\frac{2^{p_{1}-1}}{p_{1}^{p_{1}}} \frac{\eta(s)\left(\varsigma^{\prime}(s)\right)^{p_{1}}}{\mu^{p_{1}-1} s^{2\left(p_{1}-1\right)} s^{p_{1}-1}(s)}\right) \mathrm{d} s=\infty,
$$

for some $\mu \in(0,1)$, then $w^{\prime \prime}<0$.

Proof. Let $w^{\prime \prime}(\imath)>0$. From Lemmas 1 and 2, we find

$$
\frac{w\left(\phi_{i}(\imath)\right)}{w(\imath)} \geq \frac{\phi_{i}^{3}(\imath)}{\imath^{3}}
$$


and

$$
w^{\prime}(\imath) \geq \frac{\mu}{2} \imath^{2} w^{\prime \prime \prime}(\imath)
$$

Let

$$
\psi(\imath):=\varsigma(\imath) \frac{\eta(\imath)\left(w^{\prime \prime \prime}(\imath)\right)^{p_{1}-1}}{w^{p_{1}-1}(\imath)}>0 .
$$

From (12)-(14), we obtain

$$
\begin{aligned}
\psi^{\prime}(\imath) \leq & \frac{\varsigma^{\prime}(\imath)}{\zeta(\imath)} \sigma(\imath)-\varsigma(\imath) \sum_{i=1}^{j} \vartheta_{i}(\imath) \frac{\phi_{i}^{3\left(p_{1}-1\right)}(\imath)}{\imath^{3\left(p_{1}-1\right)}} w^{p_{2}-p_{1}}\left(\phi_{i}(\imath)\right) \\
& -\frac{\left(p_{1}-1\right) \mu}{2} \frac{\imath^{2}}{\varsigma^{1 / p_{1}-1}(\imath) \eta^{1 / p_{1}-1}(\imath)} \psi^{1+\left(1 /\left(p_{1}-1\right)\right)}(\imath) .
\end{aligned}
$$

Since $w^{\prime}(\imath)>0$, there exist a $\imath_{2} \geq \imath_{1}$ and a constant $M>0$ such that $w(\imath)>M$, for all $\imath \geq \imath_{2}$. Using the inequality (5) with $U=\varsigma^{\prime} / \varsigma, V=\kappa \mu \imath^{2} /\left(2 \eta^{1 / \kappa}(\imath) \varsigma^{1 / \kappa}(\imath)\right)$ and $u=\psi$, we get

$$
\psi^{\prime}(\imath) \leq-M^{p_{2}-p_{1}} \zeta(\imath) \sum_{i=1}^{j} \vartheta_{i}(\imath) \frac{\phi_{i}^{3\left(p_{1}-1\right)}(\imath)}{\imath^{3\left(p_{1}-1\right)}}+\frac{2^{p_{1}-1}}{p_{1}^{p_{1}}} \frac{\eta(\imath)\left(\zeta^{\prime}(\imath)\right)^{p_{1}}}{\mu^{p_{1}-1} \imath^{2\left(p_{1}-1\right)} \varsigma^{p_{1}-1}(\imath)} .
$$

This implies that

$$
\int_{l_{1}}^{\imath}\left(M^{p_{2}-p_{1}} \varsigma(s) \sum_{i=1}^{j} \vartheta_{i}(s) \frac{\phi_{i}^{3\left(p_{2}-1\right)}(s)}{s^{3 \kappa}}-\frac{2^{p_{1}-1}}{p_{1} p_{1}} \frac{\eta(s)\left(s^{\prime}(s)\right)^{p_{1}}}{\mu^{p_{1}-1} s^{2\left(p_{1}-1\right)} \varsigma^{p_{1}-1}(s)}\right) \mathrm{d} s \leq \psi\left(\iota_{1}\right),
$$

which contradicts (11). The proof is complete.

Theorem 2. Assume that $p_{2} \geq p_{1}$ and (11) hold, for some $\mu \in(0,1)$. If

$$
u^{\prime \prime}(\imath)+M^{p_{2}-p_{1}} \widetilde{R}(\imath) u(\imath)=0
$$

is oscillatory, then (1) is oscillatory.

Proof. Assume the contrary, that (1) has a nonoscillatory solution in $\left[\imath_{0}, \infty\right)$. Without loss of generality, we only need to be concerned with positive solutions of Equation (1). Then, there exists a $\imath_{1} \geq \imath_{0}$ such that $w(\imath)>0$ and $w\left(\phi_{i}(\imath)\right)>0$ for $\imath \geq \imath_{1}$. From Lemmas 1 and 4 , we find that

$$
w^{\prime}(\imath)>0, w^{\prime \prime}(\imath)<0 \text { and } w^{\prime \prime \prime}(\imath)>0,
$$

for $\imath \geq \imath_{2}$, where $\imath_{2}$ is sufficiently large. Now, integrating (1) from $\imath$ to $l$, we have

$$
\eta(l)\left(w^{\prime \prime \prime}(l)\right)^{p_{1}-1}=\eta(\imath)\left(w^{\prime \prime \prime}(\imath)\right)^{p_{1}-1}-\int_{\imath}^{l} \sum_{i=1}^{j} \vartheta_{i}(s) w^{p_{2}-1}\left(\phi_{i}(s)\right) \mathrm{d} s .
$$

Using Lemma 3 in [17] with (17), we obtain

$$
\frac{w\left(\phi_{i}(l)\right)}{w(l)} \geq \lambda \frac{\phi_{i}(l)}{\imath}
$$

which with (18) gives

$$
\eta(l)\left(w^{\prime \prime \prime}(l)\right)^{p_{1}-1}-\eta(l)\left(w^{\prime \prime \prime}(l)\right)^{p_{1}-1}+\lambda^{p_{2}-1} \int_{l}^{l} \sum_{i=1}^{j} \vartheta_{i}(s)\left(\frac{\phi_{i}(s)}{s}\right)^{p_{2}-1} w^{p_{1}-1}(s) \mathrm{d} s \leq 0 .
$$

It follows, by $w^{\prime}>0$, that

$$
\eta(l)\left(w^{\prime \prime \prime}(l)\right)^{p_{1}-1}-\eta(l)\left(w^{\prime \prime \prime}(l)\right)^{p_{1}-1}+\lambda^{p_{2}-1} w^{p_{1}-1}(\imath) \int_{l}^{l} \sum_{i=1}^{j} \vartheta_{i}(s)\left(\frac{\phi_{i}(s)}{s}\right)^{p_{2}-1} \mathrm{~d} s \leq 0 .
$$


Taking $l \rightarrow \infty$, we have

$$
-\eta(\imath)\left(w^{\prime \prime \prime}(\imath)\right)^{p_{1}-1}+\lambda^{p_{2}-1} w^{p_{1}-1}(\imath) \int_{\imath}^{\infty} \sum_{i=1}^{j} \vartheta_{i}(s)\left(\frac{\phi_{i}(s)}{s}\right)^{p_{2}-1} \mathrm{~d} s \leq 0,
$$

that is,

$$
w^{\prime \prime \prime}(\imath) \geq \frac{\lambda^{\left(p_{2}-1\right) /\left(p_{1}-1\right)}}{\eta^{1 /\left(p_{1}-1\right)}(\imath)} w^{\left(p_{2}-1\right) /\left(p_{1}-1\right)}(l)\left(\int_{l}^{\infty} \sum_{i=1}^{j} \vartheta_{i}(s)\left(\frac{\phi_{i}(s)}{s}\right)^{p_{2}-1} \mathrm{~d} s\right)^{1 /\left(p_{1}-1\right)} .
$$

Integrating the above inequality from $\imath$ to $\infty$, we obtain

$$
-w^{\prime \prime}(\imath) \geq \lambda^{\left(p_{2}-1\right) /\left(p_{1}-1\right)} w^{\left(p_{2}-1\right) /\left(p_{1}-1\right)}(\imath) \int_{\imath}^{\infty}\left(\frac{1}{\eta(x)} \int_{x}^{\infty} \sum_{i=1}^{j} \vartheta_{i}(s)\left(\frac{\phi_{i}(s)}{s}\right)^{p_{2}-1} \mathrm{~d} s\right)^{1 /\left(p_{1}-1\right)} \mathrm{d} x,
$$

hence

$$
w^{\prime \prime}(\imath) \leq-\widetilde{R}(\imath) w^{\left(p_{2}-1\right) /\left(p_{1}-1\right)}(\imath)
$$

Let

$$
\sigma(\imath)=\frac{w^{\prime}(\imath)}{w(\imath)}
$$

then $\sigma(\imath)>0$ for $\imath \geq \imath_{1}$, and

$$
\sigma^{\prime}(\imath)=\frac{w^{\prime \prime}(\imath)}{w(l)}-\left(\frac{w^{\prime}(\imath)}{w(l)}\right)^{2} .
$$

By using (20) and the definition of $\sigma(\imath)$, we see that

$$
\sigma^{\prime}(\imath) \leq-\widetilde{R}(\imath) \frac{w^{\left(p_{2}-1\right) /\left(p_{1}-1\right)}(\imath)}{w(\imath)}-\sigma^{2}(\imath)
$$
becomes

Since $w^{\prime}(\imath)>0$, there exists a constant $M>0$ such that $w(\imath) \geq M$, for all $\imath \geq \imath_{2}$. Then, (21)

$$
\sigma^{\prime}(\imath)+\sigma^{2}(\imath)+M^{p_{2}-p_{1}} \widetilde{R}(\imath) \leq 0,
$$

From [21], we obtain (16) is nonoscillatory if and only if there exists $\iota_{3}>\max \left\{\imath_{1}, \imath_{2}\right\}$ such that (22) holds, which is a contradiction. The theorem is proved.

Theorem 3. Let $p_{2} \geq p_{1}, \phi_{i}^{\prime}(\imath)>1$ and (11) hold, for some $\mu \in(0,1)$. If

$$
\left(\frac{1}{\phi_{i}^{\prime}(\imath)} u^{\prime}(\imath)\right)^{\prime}+M^{\left(p_{2}-1\right) /\left(p_{1}-2\right)} R(\imath) u(\imath)=0
$$

is oscillatory, then (1) is oscillatory.

Proof. From the proof of Theorem 2, we find that (18) holds. Thus, it follows from $\phi_{i}^{\prime}(\imath) \geq 0$ and $w^{\prime}(\imath) \geq 0$ that

$$
\eta(l)\left(w^{\prime \prime \prime}(l)\right)^{p_{1}-1}-\eta(\imath)\left(w^{\prime \prime \prime}(\imath)\right)^{p_{1}-1}+w^{p_{2}-1}\left(\phi_{i}(\imath)\right) \int_{\imath}^{l} \sum_{i=1}^{j} \vartheta_{i}(s) \mathrm{d} s \leq 0 .
$$

Thus, (17) becomes

$$
w^{\prime \prime}(\imath) \leq-R(\imath) w^{\left(p_{2}-1\right) /\left(p_{1}-1\right)}\left(\phi_{i}(\imath)\right) .
$$

Let

$$
\omega(\imath)=\frac{w^{\prime}(\imath)}{w\left(\phi_{i}(\imath)\right)},
$$


then $\omega(\imath)>0$ for $\imath \geq \imath_{1}$, and

$$
\begin{aligned}
\omega^{\prime}(\imath) & =\frac{w^{\prime \prime}(\imath)}{w\left(\phi_{i}(\imath)\right)}-\frac{w^{\prime}(\imath)}{w^{2}\left(\phi_{i}(\imath)\right)} w^{\prime}\left(\phi_{i}(l)\right) \phi_{i}^{\prime}(\imath) \\
& \leq \frac{w^{\prime \prime}(\imath)}{w\left(\phi_{i}(\imath)\right)}-\phi_{i}^{\prime}(\imath)\left(\frac{w^{\prime}(\imath)}{w\left(\phi_{i}(l)\right)}\right)^{2}
\end{aligned}
$$

From (25) and (26), we find

$$
\omega^{\prime}(\imath)+M^{\left(p_{2}-1\right) /\left(p_{1}-2\right)} R(\imath)+\phi_{i}^{\prime}(\imath) \omega^{2}(\imath) \leq 0 .
$$

From [21], we find (23) is nonoscillatory if and only if there exists $\imath_{3}>\max \left\{\imath_{1}, \imath_{2}\right\}$ such that (27) holds, which is a contradiction. The theorem is proved.

Corollary 2. Let $p_{2}=p_{1}$ and (11) hold. If

$$
\lim _{\imath \rightarrow \infty} \frac{1}{H\left(\imath, \imath_{0}\right)} \int_{l_{0}}^{\imath}\left(H(\imath, s) \widetilde{R}(s)-\frac{1}{4} h^{2}(\imath, s)\right) \mathrm{d} s=\infty
$$

or

$$
\liminf _{l \rightarrow \infty} \int_{\imath}^{\infty} \widetilde{R}(s) \mathrm{d} s>\frac{1}{4}
$$

then (1) is oscillatory.

Corollary 3. Let $p_{2}=p_{1}$ and (11) hold. If $\varepsilon \in(0,1 / 4]$ such that

$$
\imath^{2} \widetilde{R}(s) \geq \varepsilon
$$

and

$$
\limsup _{l \rightarrow \infty}\left(\imath^{\varepsilon-1} \int_{l_{0}}^{\imath} s^{2-\varepsilon} \widetilde{R}(s) \mathrm{d} s+\imath^{1-\widetilde{\varepsilon}} \int_{\imath}^{\infty} s^{\widetilde{\varepsilon}} \widetilde{R}(s) \mathrm{d} s\right)>1,
$$

where $\widetilde{\varepsilon}=\frac{1}{2}(1-\sqrt{1-4 \varepsilon})$, then (1) is oscillatory.

Corollary 4. Let $p_{1}=p_{2}$ and (4) holds. If

$$
\liminf _{l \rightarrow \infty} \int_{\phi(l)}^{\imath} \frac{\lambda^{p_{2}-1}}{6^{p_{2}-1}} \frac{\zeta_{l_{0}}(s) \sum_{i=1}^{j} \vartheta_{i}(s) \phi_{i}^{3\left(p_{2}-1\right)}(s)}{\zeta_{l_{0}}^{\left(p_{2}-1\right) /\left(p_{1}-1\right)}\left(\phi_{i}(s)\right) \eta^{\left(p_{2}-1\right) /\left(p_{1}-1\right)}\left(\phi_{i}(s)\right)} \mathrm{d} s>\frac{1}{\mathrm{e}^{\prime}}
$$

then (2) is oscillatory.

Corollary 5. Let $p_{1}=p_{2}$, (4) and

$$
\int_{\imath_{0}}^{\infty}\left(M^{p_{2}-p_{1}} \zeta(s) \zeta_{l_{0}}(s) \sum_{i=1}^{j} \vartheta_{i}(s) \frac{\phi_{i}^{3 \kappa}(s)}{s^{3 \kappa}}-\frac{2^{p_{1}-1}}{p_{1}^{p_{1}}} \frac{\eta(s)\left(\varsigma^{\prime}(s)\right)^{p_{1}}}{\mu^{p_{1}-1} s^{2\left(p_{1}-1\right)} \varsigma^{p_{1}-1}(s)}\right) \mathrm{d} s=\infty,
$$

hold, for some $\mu \in(0,1)$. If

$$
\lim _{l \rightarrow \infty} \frac{1}{H\left(\imath, \imath_{0}\right)} \int_{l_{0}}^{\imath}\left(H(\imath, s) \widehat{R}(s)-\frac{1}{4} h^{2}(\imath, s)\right) \mathrm{d} s=\infty
$$

or

$$
\liminf _{l \rightarrow \infty} \int_{\imath}^{\infty} \widehat{R}(s) \mathrm{d} s>\frac{1}{4}
$$

then (2) is oscillatory.

Corollary 6. Let $p_{1}=p_{2}$ and (29) hold. If $\varepsilon \in(0,1 / 4]$ such that

$$
\imath^{2} \widehat{R}(s) \geq \varepsilon
$$

and

$$
\limsup _{\imath \rightarrow \infty}\left(\imath^{\varepsilon-1} \int_{\imath_{0}}^{\imath} s^{2-\varepsilon} \widehat{R}(s) \mathrm{d} s+\imath^{1-\widetilde{\varepsilon}} \int_{\imath}^{\infty} s^{\widetilde{\varepsilon}} \widehat{R}(s) \mathrm{d} s\right)>1
$$


where $\widetilde{\varepsilon}$ is defined as in Corollary 3, then (2) is oscillatory.

Example 1. For $\imath \geq 1$, consider the equation:

$$
\left(\imath^{3}\left(w^{\prime \prime \prime}(\imath)\right)^{3}\right)^{\prime}+\frac{\vartheta_{0}}{\imath^{7}} w^{3}(\gamma \imath)=0,
$$

we see that $p_{1}=p_{2}=4, \eta(\imath)=\imath^{3}, \phi_{i}(\imath)=\gamma$ and $\vartheta(\imath)=\vartheta_{0} / \imath^{7}, \gamma \in(0,1]$ and $\vartheta_{0}>0$. Thus, we obtain

$$
\widetilde{R}(\imath)=\lambda\left(\frac{\vartheta_{0}}{6}\right)^{1 / 3} \gamma \frac{1}{2 \imath^{2}}
$$

By Corollaries 1 and 2, Equation (30) is oscillatory if

$$
\begin{gathered}
\vartheta_{0}>\frac{6^{3}}{e\left(\ln \frac{1}{\gamma}\right) \gamma^{6}}, \\
\vartheta_{0}>\left(\frac{3^{4}}{2}\right) \frac{1}{\gamma^{9}}
\end{gathered}
$$

and

$$
\vartheta_{0}>6\left(\frac{1}{4 \gamma}\right)^{3}
$$

respectively. Thus, Equation (30) is oscillatory if

$$
\vartheta_{0}>\max \left\{\left(\frac{3^{4}}{2}\right) \frac{1}{\gamma^{9}}, 6\left(\frac{1}{4 \gamma}\right)^{3}\right\}=\left(\frac{3^{4}}{2}\right) \frac{1}{\gamma^{9}} .
$$

Example 2. Consider the equation

$$
\left(\imath^{3}\left(w^{\prime \prime \prime}(\imath)\right)^{3}\right)^{\prime}+\left(w^{\prime \prime \prime}(\imath)\right)^{3}+\frac{\vartheta_{0}}{\imath^{5}} w^{3}(\imath / 2)=0, \imath \geq 1, \vartheta_{0}>0 .
$$

Let $p_{1}=p_{2}=4, \eta(\imath)=\imath^{3}, \beta(\imath)=1, \phi_{i}(\imath)=\imath / 2$ and $\vartheta(\imath)=\vartheta_{0} / \imath^{5}$. Then, it is easy to verify that

$$
\begin{aligned}
& \int_{l_{0}}^{\infty}\left[\frac{1}{\eta(s)} \exp \left(-\int_{\imath_{0}}^{s} \frac{\beta(x)}{\eta(x)} d x\right)\right]^{1 / p_{1}-1} d s \\
= & \int_{\imath_{0}}^{\infty}\left[\frac{1}{s^{3}} \exp \left(-\int_{\iota_{0}}^{s} \frac{1}{s^{3}} d x\right)\right]^{1 / 3} d s=\infty .
\end{aligned}
$$

By Corollary 5, Equation (32) is oscillatory.

\section{Conclusions}

In this work, we study the asymptotic and oscillatory properties of solutions of the fourth-order delay differential equations with $p$-Laplacian-like operators. Using the Riccati transformation, we obtained new criteria that guarantee the oscillation of all solutions of the studied equations. In future work, we will study oscillatory properties of Equation (1) under the condition

$$
\int_{l_{0}}^{\infty} \frac{1}{\eta^{1 / p_{1}-1}(s)} \mathrm{d} s<\infty
$$

An interesting problem is to extend our results to even-order damped differential equations with $p$-Laplacian-like operators

$$
\left(\eta(\imath)\left(w^{(\kappa-1)}(\imath)\right)^{p-1}\right)^{\prime}+\beta(\imath)\left|w^{\prime \prime \prime}(\imath)\right|^{p_{1}-2} w^{\prime \prime \prime}(\imath)+\vartheta(\imath) f(w(\phi(\imath)))=0 .
$$


under the condition

$$
\int_{l_{0}}^{\infty}\left[\frac{1}{\eta(s)} \exp \left(-\int_{l_{0}}^{s} \frac{\beta(x)}{\eta(x)} d x\right)\right]^{1 / p_{1}-1} d s<\infty
$$

Author Contributions: Conceptualization, O.B., F.G., J.A., K.S.A.-G. and M.A.-K.; Data curation, O.B., F.G., J.A., K.S.A.-G. and M.A.-K.; Formal analysis, O.B., F.G., J.A., K.S.A.-G. and M.A.-K.; Investigation, O.B., F.G., J.A., K.S.A.-G. and M.A.-K.; Methodology, O.B., F.G., J.A., K.S.A.-G. and M.A.-K. All authors have read and agreed to the published version of the manuscript.

Funding: This research received no external funding.

Institutional Review Board Statement: Not applicable.

Informed Consent Statement: Not applicable.

Data Availability Statement: Not applicable.

Acknowledgments: The authors thank the reviewers for their useful comments, which led to the improvement of the content of the paper. This work has been supported by the Polish National Science Centre under the grant OPUS 18 No. 2019/35/B/ST8/00980.

Conflicts of Interest: The author declare no conflict of interest.

\section{References}

1. Hale, J.K. Partial neutral functional differential equations. Rev. Roum. Math. Pures Appl. 1994, 39, 339-344.

2. MacDonald, N. Biological Delay Systems: Linear Stability Theory; Cambridge Studies in Mathematical Biology; Cambridge University Press: Cambridge, UK, 1989; Volume 8.

3. Dzrina, J.; Jadlovska, I. A note on oscillation of second-order delay differential equations. Appl. Math. Lett. 2017, 69, 126-132. [CrossRef]

4. Bohner, M.; Grace, S.R.; Jadlovska, I. Sharp oscillation criteria for second-order neutral delay differential equations. Math. Meth. Appl. Sci. 2020, 43, 10041-10053. [CrossRef]

5. Baculikova, B. Oscillation of second-order nonlinear noncanonical differential equations with deviating argument. Appl. Math. Lett. 2019, 91, 68-75. [CrossRef]

6. Baculikova, B., Dzurina, J., Graef, J. On the oscillation of higher-order delay differential equations. Math. Slovaca 2012, 187, 387-400.

7. Grace, S., Agarwal, R., Graef, J. Oscillation theorems for fourth order functional differential equations. J. Appl. Math. Comput. 2009, 30, 75-88. [CrossRef]

8. Li, T., Baculikova, B., Dzurina, J., Zhang, C. Oscillation of fourth order neutral differential equations with p-Laplacian like operators, Bound. Value Probl. 2014, 2014, 56. [CrossRef]

9. Zhang, C., Agarwal, R., Li, T. Oscillation and asymptotic behavior of higher-order delay differential equations with $\mathrm{p}$-Laplacian like operators. J. Math. Anal. Appl. 2014, 409, 1093-1106. [CrossRef]

10. Kiguradze, I. T., Chanturiya, T. A. Asymptotic Properties of Solutions of Nonautonomous Ordinary Differential Equations; Kluwer Academic Publishers: Dordrecht, The Netherlands, 1993.

11. Zhang, C., Li, T., Saker, S. Oscillation of fourth-order delay differential equations. J. Math. Sci. 2014, 201. 296-308. [CrossRef]

12. Bazighifan, O. On the oscillation of certain fourth-order differential equations with $p$-Laplacian like operator. Appl. Math. Comput. 2020, 386, 125475. [CrossRef]

13. Zhang, C., Li, T., Suna, B., Thandapani, E. On the oscillation of higher-order half-linear delay differential equations. Appl. Math. Lett. 2011, 24, 1618-1621. [CrossRef]

14. Elabbasy, E. M., Thandpani, E., Moaaz, O., Bazighifan, O. Oscillation of solutions to fourth-order delay differential equations with midlle term. Open J. Math. Sci. 2019 , 3, 191-197. [CrossRef]

15. Chatzarakis, G. E., Grace,S., Jadlovska, I., Li, T., Tunç, E. Oscillation criteria for third-order Emden-Fowler differential equations with unbounded neutral coefficients. Complexity 2019, 2019, 691758. [CrossRef]

16. Agarwal, R., Grace, S., O'Regan, D. Oscillation Theory for Difference and Functional Differential Equations; Kluwer Academic Publishers: Dordrecht, The Netherlands, 2000.

17. Bazighifan, O.; Almutairi, A.; Almarri, B.; Marin, M. An Oscillation Criterion of Nonlinear Differential Equations with Advanced Term. Symmetry 2021, 13, 843. [CrossRef]

18. Ch.G. Philos, On the existence of nonoscillatory solutions tending to zero at $\infty$ for differential equations with positive delays. Arch. Math. 1981, 36, 168-178. [CrossRef]

19. Chatzarakis, G. E., Li, T. Oscillations of differential equations generated by several deviating arguments. Adv. Differ. Equ. 2017, 2017, 1-24. [CrossRef] 
20. Chatzarakis, G. E., Li, T. Oscillation criteria for delay and advanced differential equations with nonmonotone arguments. Complexity 2018, 2018, 1-18. [CrossRef]

21. Agarwal, R., Shieh, S. L., Yeh, C. C. Oscillation criteria for second order retarde ddifferential equations. Math. Comput. Model. 1997, 26, 1-11. [CrossRef]

22. Bazighifan, O.; Dassios, I. Riccati Technique and Asymptotic Behavior of Fourth-Order Advanced Differential Equations. Mathematics 2020, 8, 590. [CrossRef]

23. Ghanim, F.; Al-Janaby, H.F.; Bazighifan, O. Some New Extensions on Fractional Differential and Integral Properties for MittagLeffler Confluent Hypergeometric Function. Fractal Fract. 2021, 5, 143. [CrossRef]

24. Philos, C. Oscillation theorems for linear differential equation of second order. Arch. Math. 1989, 53, 483-492. [CrossRef]

25. Zhang, Q., Yan, J. Oscillation behavior of even order neutral differential equations with variable coefficients. Appl. Math. Lett. 2006, 19, 1202-1206. [CrossRef]

26. Park, C.; Moaaz, O.; Bazighifan, O. Oscillation Results for Higher Order Differential Equations. Axioms 2020, 9, 14. [CrossRef]

27. Agarwal, R.P.; Bazighifan, O.; Ragusa, M.A. Nonlinear Neutral Delay Differential Equations of Fourth-Order: Oscillation of Solutions. Entropy 2021, 23, 129. [CrossRef] [PubMed]

28. Tang, S.; Li, T.; Thandapani, E. Oscillation of higher-order half-linear neutral differential equations. Demonstr. Math. 2013, 1, 101-109. [CrossRef] 